\title{
Display Name
}

National Cancer Institute

\section{Source}

National Cancer Institute. Display Name. NCI Thesaurus. Code C70896.

The standardized text associated with a code in a particular code system. 